\title{
Impact of the HIV/AIDS Epidemic on the Neurodevelopment of Preschool-Aged Children in Kinshasa, Democratic Republic of the Congo
}

\author{
Annelies Van Rie, MD, PhD ${ }^{\mathrm{a}}$, Aimee Mupuala, MD ${ }^{\mathrm{b}}$, and Anna Dow, MSPH ${ }^{\mathrm{a}}$ \\ aDepartment of Epidemiology, University of North Carolina, Chapel Hill, North Carolina \\ ${ }^{b}$ Department of Pediatrics, University of Kinshasa, Kinshasa, Democratic Republic of Congo
}

\begin{abstract}
OBJECTIVES-Pediatric HIV infection is a growing problem in most regions of the world. Data on the effects of HIV on the neurodevelopment of children in resource-poor settings are scarce but necessary to guide interventions. The purpose of this study was to compare the neurodevelopment of preschool-aged HIV-infected, HIV-affected (HIV-uninfected AIDS orphans and HIV-uninfected children whose mother had symptomatic AIDS), and healthy control children in Kinshasa, Democratic Republic of Congo.
\end{abstract}

METHODS-Thirty-five HIV-infected, $35 \mathrm{HIV-affected,} \mathrm{and} 90$ control children aged 18 to 72 months were assessed by using the Bayley Scales of Infant Development II, Peabody Developmental Motor Scales, Snijders-Oomen Nonverbal Intelligence Test, and Rossetti Infant-Toddler Language Scale, as appropriate for age.

RESULTS-Overall, 60\% of HIV-infected children had severe delay in cognitive function, $29 \%$ had severe delay in motor skills, $85 \%$ had delays in language expression, and $77 \%$ had delays in language comprehension, all significantly higher rates as compared with control children. Young HIV-infected children (aged 18-29 months) performed worse, with $91 \%$ and $82 \%$ demonstrating severe mental and motor delay, respectively, compared with $46 \%$ and $4 \%$ in older HIV-infected children (aged 30-72 months). HIV-affected children had significantly more motor and language expression delay than control children.

CONCLUSIONS-The impact of the HIV pandemic on children's neurodevelopment extends beyond the direct effect of the HIV virus on the central nervous system. AIDS orphans and HIVnegative children whose mothers had AIDS demonstrated significant delays in their neurodevelopment, although to a lesser degree and in fewer developmental domains than HIVinfected children. Young HIV-infected children were the most severely afflicted group, indicating the need for early interventions. Older children performed better as a result of a "survival effect," with only those children with less aggressive disease surviving.

\section{Keywords}

HIV; AIDS; neurodevelopment; child; Africa 
THE HIV/AIDS EPIDEMIC continues to affect millions of children in developing countries, because access to prevention of mother-to-child transmission interventions and highly active antiretroviral treatment (HAART) have not yet reached wide coverage in many high HIV prevalence countries. At the end of 2006, an estimated 2.3 million children were living with HIV/AIDS and 530000 were newly infected with HIV in 2006. The Democratic Republic of Congo (DRC) is home to an estimated 120000 children living with HIV and 680000 AIDS orphans. ${ }^{1}$

Since the first reports of pediatric AIDS in the 1980s, delayed neurodevelopment has been a well-recognized complication of HIV disease. ${ }^{2,3}$ Experiences from the United States and Europe indicate that HIV central nervous system (CNS) involvement can occur before there is significant immunosuppression and may be the first AIDS-defining illness in as many as $18 \%$ of pediatric patients. ${ }^{4,5}$ HIV-related encephalopathy can present as static or progressive HIV encephalopathy, with microcephaly, delay or loss of developmental milestones (motor, mental and language), and pyramidal tract symptoms. ${ }^{6}$ Before HAART was introduced, encephalopathy was reported in $35 \%$ to $50 \%$ of children with a diagnosis of AIDS in the United States. ${ }^{5,7-10}$ HAART can prevent and reverse encephalopathy present at the time of HAART initiation. ${ }^{11,12}$ Consequently, the introduction of HAART in the United States has reduced the incidence of encephalopathy to $<2 \% .^{11}$

Results from studies in the United States and Europe may not be generalizable to the population in Africa and other resource-poor settings, where there is also a high prevalence of malnutrition, malaria, tuberculosis and other opportunistic infections, and substantially different childrearing environments. Despite that sub-Saharan Africa is home to $80 \%$ of HIV-infected children, there is a paucity of data on neurodevelopment from this region. Results of the few studies demonstrated HIV-related CNS involvement at similar or higher prevalence rates compared with the United States. A study of Rwandan children 6 months to 2 years of age observed that $40 \%$ of HIV-infected children had an abnormal neurodevelopmental examination at 18 months compared with only $5 \%$ of HIV-exposed uninfected children, and gross motor scores were significantly lower at all studied time points in HIV-infected children. ${ }^{13}$ A study in the DRC documented developmental impairment in both asymptomatic HIV-infected children and HIV-exposed, uninfected children compared with control children, supporting the idea that there may be an environmental component compounding the delay as a result of the presence of HIV in the CNS. ${ }^{14}$ A study of Ugandan children from birth to 2 years found that HIV-infected infants not only scored lower in mental and motor development but also demonstrated greater deceleration in their rate of motor development compared with HIVexposed, uninfected infants and a control group. ${ }^{15}$ At school age (6-12 years), these HIVinfected children experienced poorer growth and more acute illness but were not significantly different in their neurologic, motor, and psychometric development compared with control children, suggesting that children with more aggressive encephalopathy may not survive to school age in the absence of HAART. ${ }^{16}$ A study of children in Tanzania found that the developmental scores decreased with increasing age, suggesting a cumulative risk for poor neurodevelopment caused by HIV, poverty, and the stress placed on families who care for HIVinfected individuals. ${ }^{17}$ This study aimed to examine the effects of the HIV epidemic on the cognitive, motor, and language development of preschool-aged children in Kinshasa, capital of the DRC.

\section{METHODS}

\section{Study Participants}

A total of 160 children aged 18 to 72 months were enrolled in the study between November 2004 and September 2005: 35 HIV-infected children; 35 uninfected, affected children; and 90 uninfected healthy control children. HIV-infected children (group 1) were identified through 
a pediatric HIV care and treatment program. Children were enrolled in the study at the time they presented to the HIV care and treatment program. Consequently, children were ARTnaive or had received $<1$ week of HAART before neurodevelopmental assessment. HIVaffected children (group 2), defined as HIV-uninfected maternal AIDS orphans or HIVuninfected children living with a mother with symptomatic AIDS, were recruited through a nongovernmental organization for social support to HIV-infected women in Kinshasa. Control children (group 3) were of HIV-uninfected healthy mothers and healthy fathers, with healthy defined as the absence of an illness that interferes with daily activities. Control children were identified at a maternity clinic with prevention of mother-to-child transmission of HIV activities. The control group was composed of 5 boys and 5 girls for every 6-month age class between 18 and 72 months. Children were excluded from the study when they presented with an acute infectious disease at the time of planned neurodevelopmental assessment. The study was approved by the institutional review boards of the Schools of Public Health of the University of North Carolina at Chapel Hill and the University of Kinshasa.

\section{Data Collection}

Clinical and anthropometric data, socioeconomic status, and health status of the parents were collected at enrollment. Socioeconomic status was assessed by inquiring about access to running water, number of bedrooms, income, type of toilet, adequacy of income, and food.

For younger children (aged 18-29 months), the Bayley Scales of Infant Development, 2nd Edition, was used to assess mental (Mental Development Index) and motor development (Psychomotor Development Index), and the Rossetti Infant-Toddler Language scale (1990 version) was used to evaluate language development. Older children (aged 30-72 months were assessed using the Peabody Developmental Motor Scales (2nd edition), the Snijders-Oomen Nonverbal (SON) Intelligence test $2 \frac{1}{2}-7$ (abridged 2004 version including situations, mosaics, categories, and patterns), and the Rossetti Infant-Toddler Language scale for children up to 36 months of age. Language development was not assessed for children who were older than 36 months. Assessment tools were translated into Lingala, the local language.

Neurodevelopmental assessment was performed by 1 pediatrician and 2 physiotherapists, who had received an 8-week neurodevelopmental assessment training (Bayley, Rossetti) in Cape Town, South Africa. In addition, the pediatrician (Dr Mupuala) had received training that consisted of 108 hours in neurodevelopmental testing procedures (Bayley, Peabody, and SON), 140 hours of neuropediatric consultations, and 72 hours of consultations at care institutions for children with neurodevelopmental disorders in Leuven, Belgium.

\section{Data Analyses}

Group means were calculated for the norm-referenced Bayley Scales of Infant DevelopmentII, Peabody, and SON. The neurodevelopment of children was classified as "no delay" when their score fell within 1 SD of the mean standardized score, (100) "moderate delay" when they scored between 1 and 2 SDs from the mean (ie, 70-84), or "severe delay" when they score <2 SDs ( $\leq 69)$. For the Rossetti test, we computed a global baseline and ceiling age level (ie, the oldest 3-month age level at which the child mastered all items in language expression and language comprehension). Children were considered to have "no delay" when their score fell within their 3-month age category or when they scored in the 3-month age category below their age. They were considered "delayed" in their language development when they scored $\geq 23$ month categories below their age.

Group means were compared by using $t$ tests or the nonparametric Wilcoxon ranked-sum tests. The $\chi^{2}$ test for contingency tables and Fisher's exact tests compared categorical and event frequency data. Analysis was conducted by using SAS 8.2 (SAS Institute, Inc, Cary, NC). 


\section{RESULTS}

\section{Demographic, Socioeconomic, and Clinical Characteristics}

The mean age of participants was 43.7 months and did not differ significantly a groupings (Table 1). Approximately $50 \%$ of children were male. None of the control children were orphans (by inclusion criteria). Among HIV-infected children, 28.6\% were maternal and 40\% were paternal orphans. Among affected children, $35.5 \%$ were paternal and $8.6 \%$ were maternal orphans. Malnutrition and stunting, defined as $z$ scores $>2$ SDs below the mean weight for age and height for age, was significantly more prevalent among HIV-infected and HIV-affected children, but even $16.7 \%$ of control children were malnourished, reflecting the high level of poverty in the DRC. HIV-affected children and HIV-infected chil-dren had a significantly poorer socioeconomic living standard compared with control children. The proportion of children aged $<60$ months with microcephaly, defined as head circumference less than the third percentile, was the highest among HIV-infected children (11.1\%), compared with $6.7 \%$ among HIV-affected children and $1.4 \%$ among control children (Table 1).

\section{Neurodevelopment of Control Children: Comparison With Normative Reference Population}

Mean standardized test scores of 70 healthy control children 18 to 29 months of age were 85.3 (SD: 18.0) for the Bayley Mental Development Index and 94.1 (SD: 13.1) for the Bayley Psychomotor Development Index. The mean standardized test scores for the 20 healthy control children 30 to 72 months of age were 84.6 (SD: 22.4) for the SON and 99.2 (SD: 7.9) for the Peabody. Compared with the normative reference population (1700 children from the United States for the Bayley, 2003 children from the Unites States for Peabody, and 1124 children from the Netherlands for SON), these mean scores were significantly lower for mental development $(P<.0001$ for Bayley Mental Development Index and SON) but similar for motor development ( $P=.08$ for Bayley Psychomotor Development Index and 0.7 for Peabody).

Both moderate (26.7\%) and severe (24.4\%) delay in mental development was observed, and mental delay was more frequent in older children. In contrast, severe motor delay was not observed among control children, and moderate motor delay was present in $7.8 \%$ and was more frequent among young children (aged 18-29 months) than older children (30-72 months), with $25 \%$ and $2.9 \%$ moderate motor delay, respectively (Table 2). Among the children tested for language development (aged 18-36 months), delay in language comprehension and language expression was found in $12.9 \%$ (Table 3 ).

\section{Mental Development in HIV-Infected and HIV-Affected Children}

Performance on the assessment of mental development was significantly poorer among HIVinfected children compared with control children (Table 2). Severe delay was present in 60\% of HIV-infected and $40 \%$ of HIV-affected children. Severe mental delay was again more frequently observed among younger HIV-infected children, with $90.9 \%$ of HIV-infected children aged 18 to 29 months presenting with severe delay compared with $46 \%$ among HIVinfected children aged 30 to 72 months.

\section{Motor Developmental in HIV-Infected and HIV-Affected Children}

Severe delay in motor development was observed in $28.6 \%$ of HIV-infected and $14.3 \%$ of HIVaffected children, and moderate delay was present in $40 \%$ of HIVinfected and $14.3 \%$ of HIVaffected children (Table 2). Motor delay was more prevalent among younger children (aged 18-29 months), with $81.8 \%$ of HIV-infected and $38.5 \%$ of HIV-affected young children presenting with severe delay, compared with only $4 \%$ of older HIV-infected and $0 \%$ of HIVaffected children. 


\section{Language}

HIV-infected children 18 to 36 months of age demonstrated significantly higher rates of delay in language expression $(84.6 \%)$ and language comprehension (76.7\%) compared with control children $(P<.0001)$. Language expression but not language comprehension was delayed in the HIV-affected children compared with the control children $(P<.02$; Table 3$)$.

\section{DISCUSSION}

Moderate to severe delay in mental, motor, and language development was observed in the majority of HIV-infected children and in a substantial proportion of HIV-affected children. Young HIV-infected children demonstrated more frequent and more severe delay in mental and motor development compared with older HIV-infected children.

The frequent delay in neurodevelopment among HIV-infected children was also observed in the previous studies of African HIV-infected children. ${ }^{13-15,18}$ Comparison of results between studies is difficult because of differences in age groups studied and the diversity of neuroassessment tools used. Two studies that also used the Bayley scales (versions I and II) observed that, by 18 months of age, severe motor delay was observed among $27 \%$ to $30 \%$ and severe mental delay in $26 \%$ to $27 \%$ of HIV-infected children. ${ }^{15,18}$

In contrast to previous studies, ${ }^{15,19-21}$ we observed that mental and not motor delay was most severely affected by HIV infection. The high prevalence of delay in cognitive development may in part be attributable to differences in child-rearing practices and lack of exposure to educational toys and multimedia by African children. This is supported by the high frequency $(24.4 \%)$ of severe delay in mental development among the healthy control children, which is much higher than a $2.2 \%$ rate that one would expect if the distribution were similar to that in the reference population.

In this study, the impact of HIV infection on both mental and motor development was most pronounced among the younger children. Although we cannot exclude the effect of using different assessment tools in the 2 age groups, we believe that the observed increased prevalence of delay in the younger HIV-infected group can most likely be attributed to the absence of access to HAART before the start of this study. In the absence of HAART, children with fast progression and more aggressive disease are likely to die before reaching the age of $2 .{ }^{22,23}$ The older children included in the study may thus represent a group of children with milder disease. A similar "survival effect" was reported from Uganda, where HIV-infected children aged 6 to 12 years were no longer different in their neurodevelopment compared with control children. ${ }^{16}$

Three other studies also assessed the development of HIV-exposed, uninfected children in subSaharan Africa. ${ }^{13-15}$ Two studies did not observe significant differences between HIVexposed, uninfected children and control children. In contrast to our study, these children were younger ( $<2$ years of age), and the socioeconomic status and quality of the home environment of HIV-exposed children was not different from that of control children, indicating that the living conditions of these children was not substantially affected by HIV. In the remaining study, HIV-exposed, uninfected children who were aged $\geq 2$ years had a significantly poorer performance on global cognitive measures but comparable motor performance. ${ }^{14}$ No data were presented on the living conditions of these children. In our study, in which children affected by AIDS in the family were selected, we observed higher frequencies of poor motor, mental, and language expression development among HIV-affected, seronegative children. The data suggest that AIDS in the family may lead to poverty, poor health, poor living conditions, and fewer positive parent- child interactions that are conducive to a child's neurodevelopment. 
Several limitations to the study should be noted. The sample size was too small to model the impact of covariates and comorbidities using logistic regression. The cross-sectional nature of this study did not allow exclusion of factors that may have had an impact on the child's neurodevelopment before study assessment. Neurologic imaging and standardized classification of neurologic examination were not available. Assessment of the language development was not available for the older children, because we were unable to adapt an existing tool for use in Lingala. Using the classification as normal, moderate, and severe neurodevelopmental delay based on US or European norms may have led to misclassification of children and biased the results to a higher prevalence of mental delay. We chose not to modify the Bayley assessment tools to make the assessment culturally appropriate because we were interested in comparisons between groups and preferred to facilitate comparability between past and future studies in other settings.

\section{CONCLUSIONS}

HIV-infected children who presented for care in Kinshasa performed poorly compared with HIV-affected and control children on assessments of motor, mental, and language development. HIV orphans and children who were living with parents who had AIDS were also at increased risk for developmental delay. Compared with control children, they achieved lower scores for motor development and tended to have slower scores for cognitive development. Our results suggest that HIV affects the neurodevelopment of children through both a direct pathway (ie, the presence of HIV in the CNS) and its effect on the child's living conditions. The high rate of developmental delay in HIV-infected and HIV-affected children as compared with control children underscores the need for screening for and prevention of neurodevelopmental delay at an early age and calls for access to early interventions and nutritional and care programs for these vulnerable children. ${ }^{24-27}$

\section{What's Known on This Subject}

In the United States, HIV encephalopathy occurred in $35 \%$ to $50 \%$ of children with AIDS before HAART. Results are not generalizable to Africa. Only 6 studies have assessed the effect of HIV on neurodevelopment in Africa, 5 of infants and 1 of school-aged children.

\section{What This Study Adds}

We demonstrate that young HIV-infected children are the most severely affected group, indicating the need for early intervention. Our study is the first to demonstrate that AIDS orphans and children who live with parents with AIDS also experience delayed neurodevelopment.

\section{Acknowledgments}

This research was supported by grant R21 MH071214 from the National Institutes of Health, Fogarty International Center and National Institute of Mental Health.

We thank Paul De Cock and Jenifer Jelsma for the training of the study team in neurodevelopmental assessment. We also thank our study team (Nadine Nossa, Iam Zephyrin, Nene Kilese, Vera Melotte, and Kashamuka Mwandagalirwa) for their efforts and the patients and families for participation in the study.

\section{Abbreviations}

HAART highly active antiretroviral treatment 
DRC Democratic Republic of Congo

CNS central nervous system

SON Snijders-Oomen Nonverbal

\section{REFERENCES}

1. World Health Organization. AIDS Epidemic Update: 2005. Geneva Switzerland: World Health Organization; 2005. United Nations programme on HIV/AIDS and World Health Organization. UNAIDS/05.19

2. Epstein LG, Sharer LR, Joshi VV, Fojas MM, Koenigsberger MR, Oleske JM. Progressive encephalopathy in children with acquired immune deficiency syndrome. Ann Neurol 1985;17:488496. [PubMed: 2988414]

3. Belman AL, Ultmann MH, Horoupian D, et al. Neurological complications in infants and children with acquired immune deficiency syndrome. Ann Neurol 1985;18:560-566. [PubMed: 3000281]

4. Vincent, J.; Bash, M.; Shanks, D.; Daigh, D.; Moriarty, R.; Fischer, G. Neurologic symptoms as the initial presentation of HIV infection in pediatric patients. Presented at: Fifth International Conference on AIDS; Montreal, Quebec, Canada. June 4-9, 1989;

5. Gabuzda DH, Hirsch MS. Neurologic manifestations of infection with human immunodeficiency virus: clinical features and pathogenesis. Ann Intern Med 1987;107:383-391. [PubMed: 3039890]

6. Belman AL, Diamond G, Dickson D, et al. Pediatric acquired immunodeficiency syndrome: neurologic syndromes. Am J Dis Child 1988;142:29-35. [PubMed: 2449072]

7. Tardieu M, Le Chenadec J, Persoz A, Meyer L, Blanche S, Mayaux MJ. HIV-1-related encephalopathy in infants compared with children and adults. French Pediatric HIV Infection Study and the SEROCO Group. Neurology 2000;54:1089-1095. [PubMed: 10720279]

8. Blanche S, Newell ML, Mayaux MJ, et al. Morbidity and mortality in European children vertically infected by HIV-1. The French Pediatric HIV Infection Study Group and European Collaborative Study. J Acquir Immune Defic Syndr Hum Retrovirol 1997;14:442-450. [PubMed: 9170419]

9. Cooper ER, Hanson C, Diaz C, et al. Encephalopathy and progression of human immunodeficiency virus disease in a cohort of children with perinatally acquired human immunodeficiency virus infection. Women and Infants Transmission Study Group. J Pediatr 1998;132:808-812. [PubMed: 9602190]

10. Lobato MN, Caldwell MB, Ng P, Oxtoby MJ. Encephalopathy in children with perinatally acquired human immunodeficiency virus infection. Pediatric Spectrum of Disease Clinical Consortium. J Pediatr 1995;126:710-715. [PubMed: 7751993]

11. Chiriboga CA, Fleishman S, Champion S, Gaye-Robinson L, Abrams EJ. Incidence and prevalence of HIV encephalopathy in children with HIV infection receiving highly active antiretroviral therapy (HAART). J Pediatr 2005;146:402-407. [PubMed: 15756229]

12. Pizzo PA, Eddy J, Falloon J, et al. Effect of continuous intra-venous infusion of zidovudine (AZT) in children with symptomatic HIV infection. N Engl J Med 1988;319:889-896. [PubMed: 3166511]

13. Msellati P, Lepage P, Hitimana DG, Van Goethem C, Van de Perre P, Dabis F. Neurodevelopmental testing of children born to human immunodeficiency virus type 1 seropositive and seronegative mothers: a prospective cohort study in Kigali, Rwanda. Pediatrics 1993;92:843-848. [PubMed: 8233747]

14. Boivin MJ, Green SD, Davies AG, Giordani B, Mokili JK, Cutting WA. A preliminary evaluation of the cognitive and motor effects of pediatric HIV infection in Zairian children. Health Psychol 1995;14:13-21. [PubMed: 7737068]

15. Drotar D, Olness K, Wiznitzer M, et al. Neuro developmental outcomes of Ugandan infants with human immunodeficiency virus type 1 infection. Pediatrics 1997;100(1) Available at: www.pediatrics.org/cgi/content/full/100/1/e5.

16. Bagenda D, Nassali A, Kalyesubula I, et al. Health, neurologic, and cognitive status of HIV-infected, long-surviving, and antiretroviral-naive Ugandan children. Pediatrics 2006;117:729-740. [PubMed: 16510653] 
17. McGrath N, Bellinger D, Robins J, Msamanga GI, Tronick E, Fawzi WW. Effect of maternal multivitamin supplementation on the mental and psychomotor development of children who are born to HIV-1-infected mothers in Tanzania. Pediatrics 2006;117(2) Available at: www.pediatrics.org/ cgi/content/full/117/2/e216.

18. McGrath N, Fawzi WW, Bellinger D, et al. The timing of mother-to-child transmission of human immunodeficiency virus infection and the neurodevelopment of children in Tanzania. Pediatr Infect Dis J 2006;25:47-52. [PubMed: 16395103]

19. Knight WG, Mellins CA, Levenson RL Jr, Arpadi SM, Kairam R. Brief report: effects of pediatric HIV infection on mental and psychomotor development. J Pediatr Psychol 2000;25:583-587. [PubMed: 11085762]

20. McNeilly LG. Communication intervention and therapeutic issues in pediatric human immunodeficiency virus. Semin Speech Lang 2000;21:63-77. [PubMed: 10768795]

21. Epstein LG, Sharer LR, Oleske JM, et al. Neurologic manifestations of human immunodeficiency virus infection in children. Pediatrics 1986;78:678-687. [PubMed: 2429248]

22. Taha TE, Graham SM, Kumwenda NI, et al. Morbidity among human immunodeficiency virus-1infected and -uninfected African children. Pediatrics 2000;106(6) Available at: www.pediatrics.org/ cgi/content/full/106/6/e77.

23. Newell ML, Coovadia H, Cortina-Borja M, Rollins N, Gaillard P, Dabis F. Mortality of infected and uninfected infants born to HIV-infected mothers in Africa: a pooled analysis. Lancet 2004;364:12361243. [PubMed: 15464184]

24. Bridge A, Kipp W, Jhangri GS, Laing L, Konde-Lule J. Nutritional status of young children in AIDSaffected households and controls in Uganda. Am J Trop Med Hyg 2006;74:926-931. [PubMed: 16687705]

25. Jin X, Sun Y, Jiang F, Ma J, Morgan C, Shen X. "Care for Development" intervention in rural China: a prospective follow-up study. J Dev Behav Pediatr 2007;28:213-218. [PubMed: 17565288]

26. Powell C, Baker-Henningham H, Walker S, Gernay J, Grantham-McGregor S. Feasibility of integrating early stimulation into primary care for undernourished Jamaican children: cluster randomised controlled trial. BMJ 2004;329:89. [PubMed: 15217841]

27. Eickmann SH, Lima AC, Guerra MQ, et al. Improved cognitive and motor development in a community-based intervention of psychosocial stimulation in northeast Brazil. Dev Med Child Neurol 2003;45:536-541. [PubMed: 12882532] 


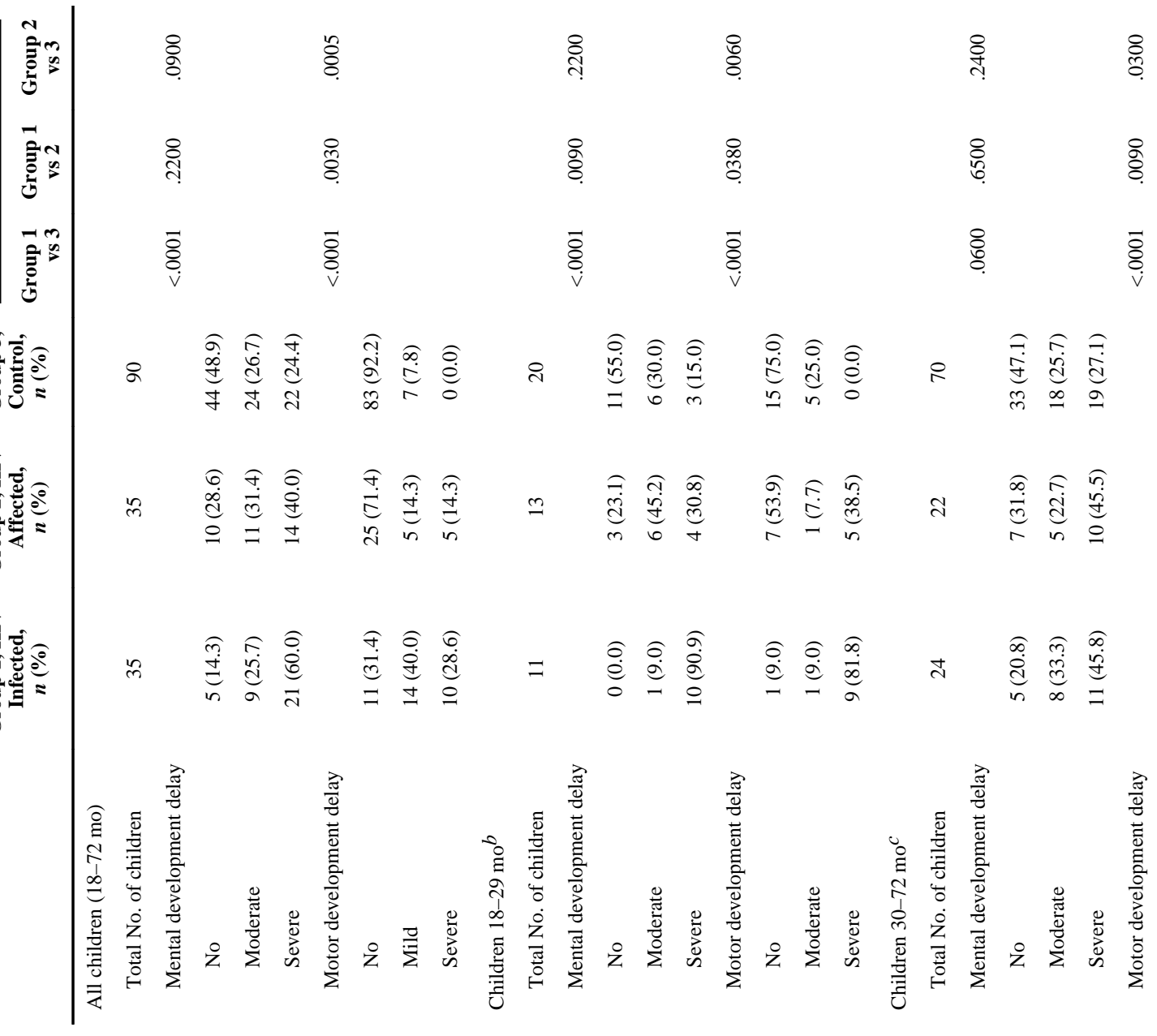




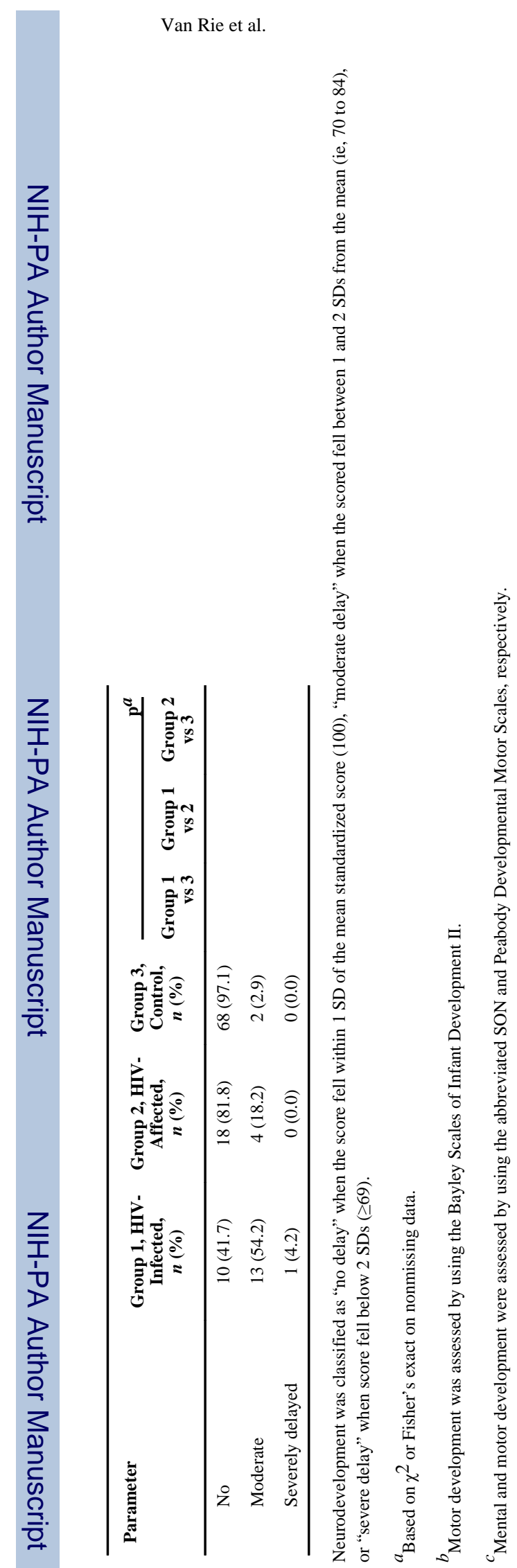

Pediatrics. Author manuscript; available in PMC 2010 July 9. 


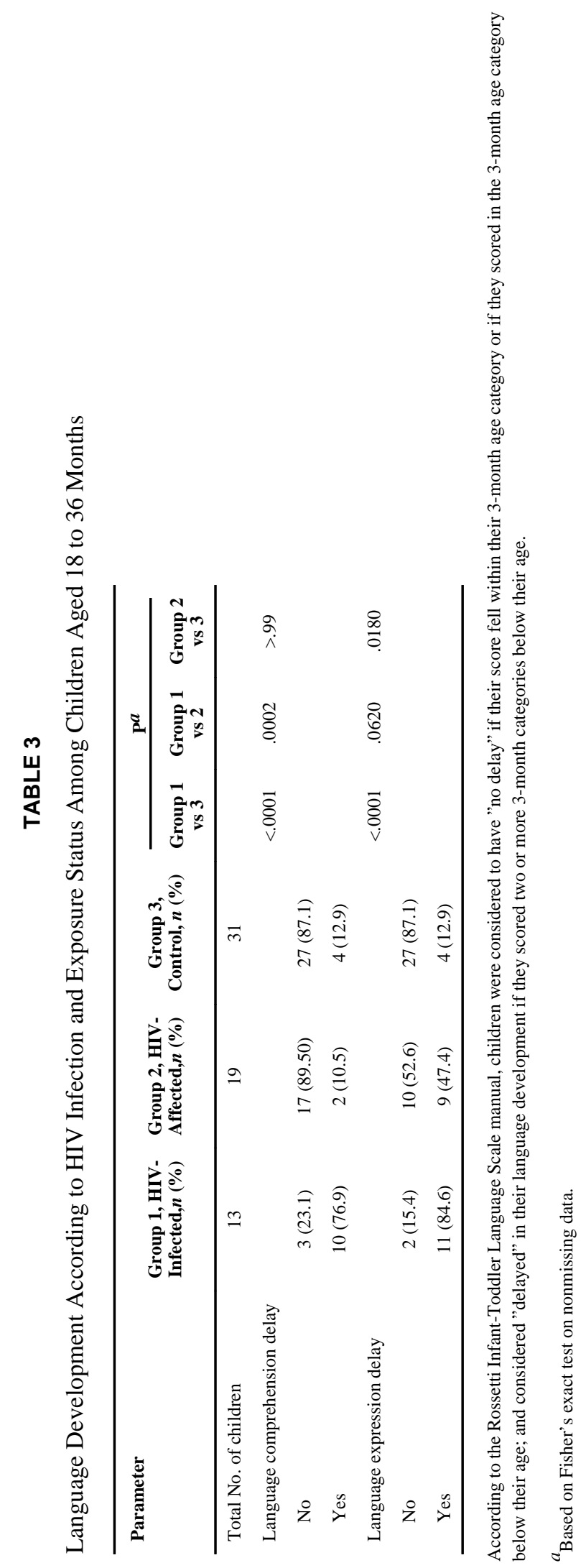

Pediatrics. Author manuscript; available in PMC 2010 July 9. 